MATHEMATICS OF COMPUTATION

Volume 72, Number 242, Pages 1003-1017

S 0025-5718(02)01443-6

Article electronically published on October 17, 2002

\title{
THE CLASS NUMBER ONE PROBLEM FOR SOME NON-ABELIAN NORMAL CM-FIELDS OF DEGREE 48
}

\author{
KU-YOUNG CHANG AND SOUN-HI KWON
}

\begin{abstract}
We prove that there is precisely one normal CM-field of degree 48 with class number one which has a normal CM-subfield of degree 16: the narrow Hilbert class field of $\mathbb{Q}(\sqrt{5}, \sqrt{101}, \theta)$ with $\theta^{3}-\theta^{2}-5 \theta-1=0$.
\end{abstract}

\section{INTRODUCTION}

According to $\mathrm{O}$ and $[\mathrm{H}]$, there exist only finitely many normal CM-fields with class number one, and their degrees are less than or equal to 436. All imaginary abelian number fields with class number one are known in $[\mathrm{Y}]$ : their degrees are less than or equal to 24 . All normal CM-fields of degree less than 48 with class number one are known by many authors ([LO1], [LO2], [Lef], LLO], [LP1], [LP2], LOO, Lou3, [P], [YPK, [PsK], [CK2, and [CK3). In the following table we sum up the numbers of the non-abelian normal CM-fields $N$ with class number one according to their degrees.

\begin{tabular}{|c|c|c|c|c|c|c|c|}
\hline$[N: \mathbb{Q}]$ & $\mathrm{nb}$ & {$[N: \mathbb{Q}]$} & $\mathrm{nb}$ & {$[N: \mathbb{Q}]$} & $\mathrm{nb}$ & {$[N: \mathbb{Q}]$} & $\mathrm{nb}$ \\
\hline 8 & 17 & 20 & 1 & 32 & 6 & 42 & 0 \\
\hline 12 & 9 & 24 & 7 & 36 & 3 & 44 & 0 \\
\hline 16 & 12 & 28 & 0 & 40 & 1 & & \\
\hline
\end{tabular}

In this paper we study the non-abelian normal CM-fields that contain a normal CM-subfield of degree 16, and will prove the following:

Theorem 1. There exists one and only one normal CM-field $N$ of degree 48 with class number one which has a normal CM-subfield of degree 16: the narrow Hilbert class field of the real dihedral number field $K_{12}=\mathbb{Q}(\sqrt{5}, \sqrt{101}, \theta)$ of degree 12 with $\theta^{3}-\theta^{2}-5 \theta-1=0$, narrow class number 4 and class number 2 . The extension $N / K_{12}$ is cyclic quartic, $d_{N}=d_{K_{12}}^{4}=2^{32} \cdot 5^{24} \cdot 101^{24}$, the maximal totally real subfield of $N$ is the Hilbert class field of $K_{12}$, and the Galois group $\operatorname{Gal}(N / \mathbb{Q})$ is isomorphic to the semi-direct product $C_{3} \rtimes D_{16}$.

\section{Prerequisite and notation}

We use the following notation. For a number field $K$, we let $h_{K}, d_{K}, \omega_{K}$, and $\zeta_{K}$ denote the class number, the absolute value of the discriminant, the number of roots of unity in $K$, and the Dedekind zeta function of $K$, respectively. If $K$

Received by the editor March 24, 2000 and, in revised form, December 26, 2000, May 2, 2001, and September 5, 2001.

2000 Mathematics Subject Classification. Primary 11R29; Secondary 11R21. 
is a CM-field, we let $h_{K}^{-}, K^{+}$and $Q_{K} \in\{1,2\}$ be the relative class number, the maximal real subfield and the Hasse unit index of $K$, respectively. For an abelian extension $F / K$ we denote by $\mathfrak{F}_{F / K}$ the finite part of its conductor. For a positive integer $n$ we let $\zeta_{n}=e^{2 i \pi / n}$. Before starting the proof of Theorem 1 we recall the well-known results which will be used later in this paper.

Proposition 1. (1) ([LOO Lemma 2]) If $K$ is a normal CM-field, then the complex conjugation is in the center of its Galois group.

(2) ([CH Lemma 13.5]) Let $K$ be a CM-field. If there is at least one ramified prime ideal in $K / K^{+}$which is lying above an odd prime, then $Q_{K}=1$.

(3) ([o, Theorem 5], $\mathrm{Ok}$, or [LOO, Theorem 5]) Let $k \subset K$ be two CM-fields. Then $h_{k}^{-}$divides $4 h_{K}^{-}$. Moreover, if $[K: k]$ is odd, then $h_{k}^{-}$divides $h_{K}^{-}$and $Q_{k}=Q_{K}$.

(4) ([LO2] and [Lou1, Proposition 6]) Let $K$ be a CM-field and let $t$ be the number of prime ideals of $K^{+}$that are ramified in the quadratic extension $K / K^{+}$. Then $2^{t-1}$ divides $h_{K}^{-}$. Moreover, if $Q_{K}=2$, then $2^{t}$ divides $h_{K}^{-}$.

(5) ([Lou1, Proposition 13] and [LO2, Proposition 2]) Let $K=L_{1} L_{2}$ be a CM-field which is a compositum of two CM-fields $L_{1}$ and $L_{2}$ with the same maximal totally real subfield. Then

$$
h_{K}^{-}=\frac{Q_{K}}{Q_{L_{1}} Q_{L_{2}}} \frac{\omega_{K}}{\omega_{L_{1}} \omega_{L_{2}}} h_{L_{1}}^{-} h_{L_{2}}^{-}
$$

and $h_{L_{1}}^{-} h_{L_{2}}^{-}$divides $4 h_{K}^{-}$. In particular, if $L_{1}$ and $L_{2}$ are isomorphic, then $\omega_{K}=\omega_{L_{1}}=\omega_{L_{2}}=2$ and $h_{K}^{-}=\left(Q_{K} / 2\right)\left(h_{L_{1}}^{-} / Q_{L_{1}}\right)^{2}$.

(6) ([M, Corollary 2.2 and 2.3]) Let $E / F$ be an extension of number fields. Then $h_{F}$ divides $[E: F] h_{E}$. Moreover, if no nontrivial abelian subextension of $E / F$ is unramified over $F$, then $h_{F}$ divides $h_{E}$.

(7) Let $K=L_{1} L_{2}$ be a $C M$-field which is a compositum of two $C M$-fields $L_{1}$ and $L_{2}$ with the same maximal totally real subfield $L_{1}^{+}=L_{2}^{+}$. If $h_{K}=1$, then $h_{L_{1}}$ and $h_{L_{2}}$ are 1 or 2 . If $h_{K}=1$ and $h_{L_{1}}=h_{L_{2}}=2$, then $h_{L_{1}^{+}}=h_{L_{2}^{+}}=2$.

Proof. We only need to prove the last statement of (7). If $h_{K}=1$ and $h_{L_{1}}=h_{L_{2}}=$ 2 , then $K$ is the Hilbert class field of $L_{1}$ and is at the same time that of $L_{2}$. Hence, $K^{+}$is the Hilbert class field of $L_{1}^{+}=L_{2}^{+}$(see [P, Lemma 6.2]).

Proposition 2. Let $K$ be a $C M$-field of degree $2 n$.

(1) $([\mathrm{W}]) h_{K}^{-}=Q_{K} \omega_{K} /(2 \pi)^{n} \cdot \sqrt{d_{K} / d_{K^{+}}} \cdot \operatorname{Res}_{s=1}\left(\zeta_{K}\right) / \operatorname{Res}_{s=1}\left(\zeta_{K^{+}}\right)$

(2) ([LO2, Proposition 9]) Let $\beta_{K}=1-\left(2 / \log d_{K}\right)$ and

$$
\varepsilon_{K}=\max \left(1-2 \pi n e^{1 / n} / d_{K}^{1 / 2 n}, 2 / 5 \exp \left(-2 \pi n / d_{K}^{1 / 2 n}\right)\right) .
$$

If $\zeta_{K}\left(\beta_{K}\right) \leq 0$, then $\operatorname{Res}_{s=1}\left(\zeta_{K}\right) \geq 2 \varepsilon_{K} /\left(e \log d_{K}\right)$.

(3) ([Lou2]) There exists a computable constant $\mu_{k}>0$ such that for any abelian extension $K / k$ of degree $m$ unramified at all the infinite places we have

$$
\operatorname{Res}_{s=1}\left(\zeta_{K}\right) \leq\left(\operatorname{Res}_{s=1}\left(\zeta_{k}\right)\right)^{m}\left(\frac{1}{2(m-1)} \log \left(d_{K} / d_{k}^{m}\right)+2 \mu_{k}\right)^{m-1} .
$$

Let $C_{m}$ denote the cyclic group of order $m, D_{m}$ the dihedral group of order $m$, $Q_{m}$ the quaternion group of order $m$ and set $G_{6}=\langle b, c, z| b^{4}=c^{2}=z^{2}=1, c^{-1} b c=$ $b z, b z=z b, c z=z c\rangle$ and $G_{9}=\langle b, c, z| b^{2}=c^{2}=z^{4}=1, c^{-1} b c=b z^{2}, b z=z b, c z=$ $z c\rangle$ (in the notation of [JL]). Throughout this paper, $N$ denotes a non-abelian 
normal CM-field of degree 48. We assume that the 3-Sylow subgroup of its Galois group $\operatorname{Gal}(N / Q)$ is normal, and we let $M$ denote the normal CM-subfield of degree 16 of $N$. According to Proposition 1, if $h_{N}=1$, then $h_{M}^{-}=1$ (moreover, either $N / M$ is ramified at least one finite place and $h_{M}=1$ or $N / M$ is unramified at all places, $h_{M}=3$, and $N$ is the Hilbert class field of $M$ ). Now, there are 26 normal CM-fields of degree 16 with relative class number one (see [LO2], Lou3], CK1], PK], and Theorem 2 below). If $\operatorname{Gal}(M / Q)$ is non-abelian, then it is equal to $Q_{8} \times C_{2}, G_{6}, D_{16}, G_{9}, D_{8} \times C_{2}$. For proving Theorem 1, we first prove that if $\operatorname{Gal}(M / Q) \neq D_{16}, G_{9}, D_{8} \times C_{2}$, then we can use Proposition 1 and the known solutions to various (relative) class number problems for suitable CM-subfields of $N$ to prove that $h_{N}>1$. Now, assume that $\operatorname{Gal}(M / Q)=D_{16}, G_{9}$, or $D_{8} \times C_{2}$. We will show that we can find a subfield $L$ of $M^{+}$such that $N / L$ is abelian and such that the use of abelian $L$-functions to factorize $\zeta_{N} / \zeta_{L}$ readily yields $\left(\zeta_{N} / \zeta_{L}\right)(s) \geq 0$ for $0<s<1$. Since $M$ is known, $L$ also is known, we will check that $\zeta_{L}(s) \leq 0$ for $0<s<1$ and we will therefore deduce that $\zeta_{N}(s) \leq 0$ for $0<s<1$. Using Proposition 2, we will obtain explicit lower bounds for $h_{N}^{-}$, according to which we will be able to compute explicit upper bounds on $d_{N}$ when $h_{N}=1$ and to construct a short list of number fields $N$ containing all such $N$ 's with $h_{N}=1$. We will finally explain how one can use the method expounded in [Lou5] and [Lou6] to compute the relative class numbers of these finitely many CM-fields $N$ that remain, thus completing the proof of Theorem 1 .

\section{CASE 1: $M$ IS ABELIAN}

We will show the following.

Proposition 3. If $N$ contains an abelian number field $M$ of degree 16 , then $h_{N}>1$.

Proof. Let $K_{3}$ be any cubic subfield of $N$. Since $N$ is non-abelian, $K_{3}$ is not normal, its normal closure $K_{6}$ is a dihedral real sextic field, and we let $k_{2}$ denote the (real) quadratic subfield of $K_{6}$. The Galois group $\operatorname{Gal}(M / \mathbb{Q})$ is isomorphic to $C_{16}, C_{8} \times C_{2}, C_{4} \times C_{4}, C_{4} \times C_{2} \times C_{2}$, or $C_{2} \times C_{2} \times C_{2} \times C_{2}$.

(i) If $\operatorname{Gal}(M / \mathbb{Q})=C_{16}$, then $\operatorname{Gal}(N / \mathbb{Q})$ is isomorphic to $C_{3} \rtimes C_{16}=\langle a, b| a^{3}=$ $\left.b^{16}=1, b^{-1} a b=a^{-1}\right\rangle$, and $N$ is a compositum of $M$ and the real dihedral field of degree 6 that is fixed by $\left\langle b^{2}\right\rangle$. According to [Lou4, Theorem 5] we have $h_{N}^{-}>1$.

(ii) If $\operatorname{Gal}(M / \mathbb{Q})=C_{8} \times C_{2}$ with $\operatorname{Gal}\left(M^{+} / \mathbb{Q}\right)=C_{8}$, then $\operatorname{Gal}(N / \mathbb{Q})=\langle a, b, c| a^{3}=$ $\left.b^{8}=c^{2}=1, b^{-1} a b=a^{-1}, a c=c a, b c=c b\right\rangle$ with $\operatorname{Gal}\left(N / N^{+}\right)=\left\langle b^{4} c\right\rangle$. The subfield $K_{12}$ fixed by $\left\langle b^{2} c\right\rangle$ is a normal CM-field with Galois group isomorphic to $Q_{12}$. By [LP1 $h_{K_{12}}^{-}>4$, whence $h_{N}^{-}>1$ by Proposition 1(3). If $\operatorname{Gal}(M / \mathbb{Q})=C_{8} \times C_{2}$ with $\operatorname{Gal}\left(M^{+} / \mathbb{Q}\right)=C_{4} \times C_{2}$, then $h_{N}^{-}>1$ by [CK1]. Hence $h_{N}^{-}>1$ by Proposition 1(3).

(iii) If $\operatorname{Gal}(M / \mathbb{Q})=C_{4} \times C_{4}$, then $\operatorname{Gal}(N / \mathbb{Q})=Q_{12} \times C_{4}$ and $\operatorname{Gal}\left(N^{+} / \mathbb{Q}\right)$ is isomorphic to either $S_{3} \times C_{4}$ or $Q_{12} \times C_{2}$. Let $\psi_{1}$ and $\psi_{2}$ be two odd primitive characters of order 4 such that $M$ is associated with the group $\left\langle\psi_{1}, \psi_{2}\right\rangle$. If $k_{2}$ is associated with $\left\langle\psi_{1}^{2}\right\rangle$ or $\left\langle\psi_{2}^{2}\right\rangle$, then $\operatorname{Gal}\left(N^{+} / \mathbb{Q}\right)=S_{3} \times C_{4}$. Assume that $k_{2}$ is associated with $\left\langle\psi_{1}^{2}\right\rangle$. Let $M_{12,1}$ be the compositum of $K_{6}$ and the quartic field associated with $\left\langle\psi_{1}\right\rangle$, and $M_{12,2}$ the compositum of $K_{6}$ and the quartic field associated with $\left\langle\psi_{1} \psi_{2}^{2}\right\rangle$. Then $M_{12,1}$ and $M_{12,2}$ are quaternion CM-fields 
of degree 12 with the same maximal real subfield $K_{6}$. According to [LP1. Theorem 1], there is no pair of $\left(M_{12,1}, M_{12,2}\right)$ such that $h_{M_{12,1}}^{-}\left|4, h_{M_{12,2}}^{-}\right| 4$, and at the same time $M_{12,1}^{+}=M_{12,2}^{+}$, whence $h_{N}^{-}>1$. By symmetry, if $k_{2}$ is associated with $\left\langle\psi_{2}^{2}\right\rangle$, then $h_{N}^{-}>1$. Assume now that $k_{2}$ is associated with $\left\langle\psi_{1}^{2} \psi_{2}^{2}\right\rangle$. Let $M_{24,1}$ be the compositum of $K_{6}$ and the imaginary cyclic quartic field associated with $\left\langle\psi_{1}\right\rangle$, and $M_{24,2}$ the compositum of $K_{6}$ and the imaginary cyclic quartic field associated with $\left\langle\psi_{2}\right\rangle$. Then $M_{24,1}$ and $M_{24,2}$ are normal CM-fields with Galois group isomorphic to $S_{3} \times C_{4}$ which have the same maximal real subfield. Using Proposition 1(5) we verify that $h_{N}^{-}=$ $h_{M_{24,1}}^{-} h_{M_{24,2}}^{-}$. By $[\mathrm{P}$ Theorem 1] there is only on CM-field of relative class number one with Galois group isomorphic to $S_{3} \times C_{4}$, whence $h_{N}^{-}>1$.

(iv) If $\operatorname{Gal}(M / \mathbb{Q})=C_{4} \times C_{2} \times C_{2}$ with $\operatorname{Gal}\left(M^{+} / \mathbb{Q}\right)=C_{4} \times C_{2}$, then $\operatorname{Gal}(N / \mathbb{Q})$ is isomorphic to either $Q_{12} \times C_{2} \times C_{2}$ or $S_{3} \times C_{2} \times C_{4}$. Let $\psi$ be the odd primitive Dirichlet character of order 4 , and let $\chi_{1}$ and $\chi_{2}$ be two quadratic odd characters such that $M$ is associated with the group $\left\langle\psi, \chi_{1}, \chi_{2}\right\rangle$. If $k_{2}$ is associated with $\left\langle\psi^{2}\right\rangle$, then the compositum $M_{12,1}$ of $K_{6}$ and the field associated with $\langle\psi\rangle$, and the compositum $M_{12,2}$ of $K_{6}$ and the field associated with $\left\langle\psi \chi_{1} \chi_{2}\right\rangle$ are normal CM-fields with Galois group $Q_{12}$ and $M_{12,1}^{+}=M_{12,2}^{+}$. By [LP1, Theorem 1], $h_{N}^{-}>1$. If $k_{2}$ is associated with $\left\langle\psi^{2} \chi_{1} \chi_{2}\right\rangle$ or $\left\langle\chi_{1} \chi_{2}\right\rangle$, then we let $M_{24,1}$ be the compositum of $K_{6}$ and the field associated with $\langle\psi\rangle$, and $M_{24,2}$ the compositum of $K_{6}$ and the field associated with $\left\langle\psi^{2}, \chi_{1}, \chi_{2}\right\rangle$. Then $\operatorname{Gal}\left(M_{24,1} / \mathbb{Q}\right)=S_{3} \times C_{4}, \operatorname{Gal}\left(M_{24,2} / \mathbb{Q}\right)=S_{3} \times C_{2} \times C_{2}, M_{24,1}^{+}=M_{24,2}^{+}$, and $N=M_{24,1} M_{24,2}$. By [P, Theorem 1] $h_{M_{24,1}}^{-}>1$ and $h_{M_{24,2}}^{-}>1$, whence according to Proposition 1(7) we have $h_{N}>1$.

(v) If $\operatorname{Gal}(M / \mathbb{Q})=C_{4} \times C_{2} \times C_{2}$ with $\operatorname{Gal}\left(M^{+} / \mathbb{Q}\right)=C_{2} \times C_{2} \times C_{2}$, then $h_{M}^{-}>1$ by CK1. Hence $h_{N}^{-}>1$.

(vi) If $\operatorname{Gal}(M / \mathbb{Q})=C_{2} \times C_{2} \times C_{2} \times C_{2}$, then $h_{M}^{-}>1$ by CK1. Hence $h_{N}^{-}>1$.

\section{4. $\operatorname{CAsE} 2: \operatorname{Gal}(M / \mathbb{Q}) \in\left\{D_{16}, Q_{8} \times C_{2}\right\}$}

In this section we assume that $\operatorname{Gal}(M / \mathbb{Q}) \in\left\{D_{16}, Q_{8} \times C_{2}\right\}$ and $h_{M}^{-}=1$. We will prove that there is exactly one field $N$ with $h_{N}=1$. In subsection 4.1 we assume that $G(M / \mathbb{Q})=D_{16}$, and in subsection 4.2 we assume that $G(M / \mathbb{Q})=Q_{8} \times C_{2}$.

4.1. $G(M / \mathbb{Q})=D_{16}$. There are five dihedral CM-fields $M$ of degree 16 with relative class number one [LO2 Theorem 10]: the narrow Hilbert class fields of $\mathbb{Q}(\sqrt{p q})$ with $(p, q) \in\{(2,257),(5,101),(5,181),(13,53),(13,61)\}$. The narrow Hilbert class field of $\mathbb{Q}(\sqrt{2 \cdot 257})$ has class number three and the remaining four $M$ 's have class number one. We set $K=\mathbb{Q}(\sqrt{p}, \sqrt{q})$ and $k=\mathbb{Q}(\sqrt{p q})$. The field $M$ has three quadratic subfields $L_{1}, L_{2}$, and $k$ with $\operatorname{Gal}\left(M / L_{1}\right)=\operatorname{Gal}\left(M / L_{2}\right)=D_{8}$, and $\operatorname{Gal}(M / k)=C_{8}$. Therefore, the Galois group $\operatorname{Gal}(N / \mathbb{Q})$ is isomorphic to $D_{16} \times C_{3}$ if $N$ contains only one cubic cyclic subfield, $D_{48}$ or $\left(D_{8} \times C_{3}\right) \rtimes^{1} C_{2}=C_{3} \rtimes D_{16}$ $=\left\langle a, b, c \mid a^{3}=b^{8}=c^{2}=1, c^{-1} b c=b^{-1}, b^{-1} a b=a^{-1}, a c=c a\right\rangle$, otherwise. In [Lef] it is proved that if $\operatorname{Gal}(N / \mathbb{Q})=D_{48}$, then $h_{N}^{-}>1$. We deal with the fields $N$ with $\operatorname{Gal}(N / \mathbb{Q})=D_{16} \times C_{3}$ in 4.1 .1 and the fields $N$ with $\operatorname{Gal}(N / \mathbb{Q})=\left(D_{8} \times C_{3}\right) \rtimes^{1} C_{2}$ in 4.1 .2 , respectively. 
4.1.1. $\operatorname{Gal}(N / \mathbb{Q})=D_{16} \times C_{3}$. Let $K_{3}$ denote the cyclic cubic subfield of $N$. Since $K_{3} / \mathbb{Q}$ is ramified, $N / M$ is ramified and if $h_{N}=1$, then $h_{M}=1$ by point (6) of Proposition 1. Therefore, $M$ cannot be equal to the narrow Hilbert class field of $\mathbb{Q}(\sqrt{2 \cdot 257})$. Note that $N / k$ is cyclic of degree 24 .

Lemma 1. Let $\chi$ be any one of the eight characters of order 24 associated with the cyclic extension $N / k$.

(1) We have $\left(\zeta_{N} / \zeta_{K}\right)(s) \geq 0$ in the range $0<s<1$.

(2) For each given $M$ with $h_{M}=1$ we can compute a bound $N_{k / \mathbb{Q}}(\mathfrak{F}) \leq C$ on the norms of the conductors $\mathfrak{F}$ of the cyclic cubic extensions $k K_{3} / k$ for the $N$ 's such that $h_{N}^{-}=1$. These bounds are listed in Table 1.

(3) Assume that $h_{N}=1$. Then $\mathfrak{F}=(l)$ for some prime $l$ which splits in $k$, or $\mathfrak{F}=\mathfrak{B}_{l}$ for some prime ideal $\mathfrak{B}_{l}$ of $k$ above a prime $l$ ramified in $k$.

(4) $h_{M}^{-}$divides $h_{N}^{-}, L(0, \chi) \in \mathbb{Q}(\sqrt{2}, \sqrt{-3})$, and $h_{N}^{-} / h_{M}^{-}=N_{\mathbb{Q}(\sqrt{2}, \sqrt{-3})}\left(\frac{1}{4} L(0, \chi)\right)^{2}$ is a perfect square which can be computed using the techniques developed in [Lou5] and Lou6].

Proof. (1) It follows from $\left(\zeta_{N} / \zeta_{K}\right)(s)=\prod_{i=1}^{11}\left|L\left(s, \chi^{i}\right)\right|^{2}$.

(2) We have verified that for the above four $M$ 's, $\zeta_{K}(s) \leq 0$ in the range $0<$ $s<1$. Hence, $\zeta_{N}(s) \leq 0$ for $0<s<1$. Using [Lou2, Lemma 12 and Proposition 13] we compute explicitly $\mu_{k} \operatorname{Res}_{s=1}\left(\zeta_{k}\right)$ and apply Proposition 2 to get lower bound for $h_{N}^{-}$. Since $M / M^{+}$is unramified at all finite places and $Q_{M}=\omega_{M}=2, N / N^{+}$is unramified at all finite places, $d_{N^{+}}=d_{k}^{12} N_{k / \mathbb{Q}}(\mathfrak{F})^{8}$, and $Q_{N}=\omega_{N}=2$. From this lower bound for $h_{N}^{-}$we obtain the upper bounds $C$ on $N_{k / \mathbb{Q}}(\mathfrak{F})$ such that $h_{N}^{-}=1$ implies $N_{k / \mathbb{Q}}(\mathfrak{F}) \leq C$.

(3) If the number of ramified primes in $K_{3} / \mathbb{Q}$ is greater than one, then 3 divides $h_{K_{3}}$, whence $3 \mid h_{N^{+}}$by Proposition 1(6). If there is a prime divisor $l$ of $N_{k / \mathbb{Q}}(\mathfrak{F})$ which is inert in $k$, then $3^{4}$ divides $h_{N}^{-}$. Since $M$ is the narrow Hilbert class field of $k,(l)$ splits completely in $M / k$, whence there are at least 4 prime ideals ramified in $N^{+} / M^{+}$which split at the same time in $M / M^{+}$. Hence, $3^{4} \mid h_{N}^{-}$by [LOO, Proposition 8].

(4) According to [Lou6], the value $L(0, \chi)$ is an algebraic integer of $\mathbb{Q}\left(\zeta_{24}\right)$ and

$$
h_{N}^{-} / h_{M}^{-}=N_{\mathbb{Q}\left(\zeta_{24}\right) / \mathbb{Q}}\left(\frac{1}{4} L(0, \chi)\right) .
$$

Let $\operatorname{Gal}(N / \mathbb{Q})=\left\langle a, b, c \mid a^{3}=b^{8}=c^{2}=1, b^{-1} a b=a, c^{-1} a c=a, c^{-1} b c=b^{-1}\right\rangle$, where $\operatorname{Gal}(N / k)=\langle a, b\rangle$. The restriction of $c$ to $k$ generates $\operatorname{Gal}(k / \mathbb{Q})$ and using Artin's reciprocity theorem we obtain that $\chi \circ c=\chi^{7}$ and

$$
\sigma_{7}(L(0, \chi))=L\left(0, \chi^{7}\right)=L(0, \chi \circ c)=L(0, \chi) .
$$

Therefore, $L(0, \chi) \in \mathbb{Q}(\sqrt{2}, \sqrt{-3})$, the subfield of $\mathbb{Q}\left(\zeta_{24}\right)$ fixed by $\sigma_{7}$. It follows that

$$
h_{N}^{-} / h_{M}^{-}=\left(N_{\mathbb{Q}(\sqrt{2}, \sqrt{-3}) / \mathbb{Q}}\left(\frac{1}{4} L(0, \chi)\right)\right)^{2}
$$

is a perfect square.

We verify that $h_{N}^{-}>1$ for all fields $N$ satisfying points (2) and (3) in Lemma 1 , and sum up the computational results in Table 1. 
TABLE 1.

\begin{tabular}{|c|c|c|c|c|}
\hline$k$ & $\mu_{k} \operatorname{Res}_{s=1}\left(\zeta_{k}\right) \leq$ & $N_{k / \mathbb{Q}}(\mathfrak{F}) \leq$ & $\mathfrak{F}$ & $h_{N}^{-}$ \\
\hline $\mathbb{Q}(\sqrt{5 \cdot 101})$ & 2.160235 & 36000 & $\begin{array}{c}(7) \\
(9) \\
(19) \\
(31) \\
(67) \\
(73) \\
(79) \\
(103) \\
(127) \\
(163) \\
(181) \\
\end{array}$ & $\begin{array}{c}193^{2} \\
463^{2} \\
9337^{2} \\
49252^{2} \\
4540144^{2} \\
1987279^{2} \\
3111696^{2} \\
8589721^{2} \\
51439012^{2} \\
180487972^{2} \\
330579292^{2}\end{array}$ \\
\hline $\mathbb{Q}(\sqrt{5 \cdot 181})$ & 2.154802 & 4700 & $\begin{array}{c}(7) \\
\mathfrak{P}_{181}\end{array}$ & $\begin{array}{c}964^{2} \\
4032^{2} \\
\end{array}$ \\
\hline $\mathbb{Q}(\sqrt{13 \cdot 53})$ & 1.976174 & 6000 & $\begin{array}{l}\mathfrak{P}_{13} \\
(19) \\
(31) \\
(43) \\
(67) \\
(73) \\
\end{array}$ & $\begin{array}{c}28^{2} \\
23863^{2} \\
130729^{2} \\
538657^{2} \\
8539888^{2} \\
11762791^{2} \\
\end{array}$ \\
\hline $\mathbb{Q}(\sqrt{13 \cdot 61})$ & 2.531191 & 18000 & $\begin{array}{c}\mathfrak{P}_{13} \\
(7) \\
(9) \\
(31) \\
(37) \\
\mathfrak{P}_{61} \\
(67) \\
(103) \\
(127)\end{array}$ & $\begin{array}{c}63^{2} \\
964^{2} \\
1519^{2} \\
189724^{2} \\
540127^{2} \\
487^{2} \\
4314289^{2} \\
22194844^{2} \\
73351108^{2}\end{array}$ \\
\hline
\end{tabular}

4.1.2. $\operatorname{Gal}(N / \mathbb{Q})=\left(D_{8} \times C_{3}\right) \rtimes^{1} C_{2}$. The field $N$ has three non-normal cubic subfields. Let $K_{3}$ be any one of them, $K_{6}$ its normal closure, and $k_{2}$ the quadratic subfield of $K_{6}$. Since if $\operatorname{Gal}\left(M / k_{2}\right)=C_{8}$, then $\operatorname{Gal}(N / \mathbb{Q})=D_{48}$ and $h_{N}^{-}>1$. It follows that $k_{2}=\mathbb{Q}(\sqrt{p})$ or $\mathbb{Q}(\sqrt{q})$, and $\operatorname{Gal}\left(M / k_{2}\right)=D_{8}$.

Lemma 2. (1) We have $\left(\zeta_{N} / \zeta_{K}\right)(s) \geq 0$ in the range $0<s<1$.

(2) There exists some positive integer $f \geq 1$ such that $\mathfrak{F}_{K_{6} / k_{2}}=(f)$. For each given $M$ with $h_{M}^{-}=1$ we can compute a bound $f \leq C$ on the conductors $(f)$ of the cyclic cubic extensions $K_{6} / k_{2}$ for the $N$ 's such that $h_{N}^{-}=1$. These bounds and the possible $f$ 's are given in Table 2.

(3) Let $\chi$ be any one of the four characters of order 12 associated with the cyclic extension $N / K$. Then $h_{M}^{-}$divides $h_{N}^{-}, L(0, \chi) \in \mathbb{Q}$, and $h_{N}^{-} / h_{M}^{-}=$ $(L(0, \chi) / 16)^{4}$ is a perfect fourth power which can be computed by using the techniques developed in [Lou5] and [Lou6]. 
TABLE 2 .

\begin{tabular}{|c|c|c|c|c|c|}
\hline$K$ & $k_{2}$ & $\mu_{k_{2}} \operatorname{Res}_{s=1}\left(\zeta_{k_{2}}\right)<$ & $f<$ & $f$ & $h_{N}^{-}$ \\
\hline $\mathbb{Q}(\sqrt{2}, \sqrt{257})$ & $\mathbb{Q}(\sqrt{257})$ & & & 1 & $2^{4}$ \\
\hline \multirow[t]{2}{*}{$\mathbb{Q}(\sqrt{5}, \sqrt{101})$} & $\mathbb{Q}(\sqrt{5})$ & 0.043633 & 13 & None & \\
\hline & $\mathbb{Q}(\sqrt{101})$ & 0.562340 & 22 & 2 & 1 \\
\hline \multirow[t]{2}{*}{$\mathbb{Q}(\sqrt{5}, \sqrt{181})$} & $\mathbb{Q}(\sqrt{5})$ & & 105 & None & \\
\hline & $\mathbb{Q}(\sqrt{181})$ & 0.855096 & 30 & 17 & $166^{4}$ \\
\hline \multirow[t]{3}{*}{$\mathbb{Q}(\sqrt{13}, \sqrt{53})$} & $\mathbb{Q}(\sqrt{13})$ & 0.146745 & 19 & $2 \cdot 5$ & $35^{4}$ \\
\hline & \multirow[t]{2}{*}{$\mathbb{Q}(\sqrt{53})$} & \multirow[t]{2}{*}{0.407728} & \multirow[t]{2}{*}{52} & $2 \cdot 5$ & $50^{4}$ \\
\hline & & & & $2 \cdot 13$ & $31^{4}$ \\
\hline \multirow[t]{5}{*}{$\mathbb{Q}(\sqrt{13}, \sqrt{61})$} & \multirow[t]{2}{*}{$\mathbb{Q}(\sqrt{13})$} & & \multirow[t]{2}{*}{130} & 11 & $85^{4}$ \\
\hline & & & & $2 \cdot 61$ & $175^{4}$ \\
\hline & \multirow[t]{3}{*}{$\mathbb{Q}(\sqrt{61})$} & \multirow[t]{3}{*}{0.487910} & \multirow[t]{3}{*}{95} & 13 & $6^{4}$ \\
\hline & & & & $2 \cdot 9$ & $142^{4}$ \\
\hline & & & & $2 \cdot 11$ & $233^{4}$ \\
\hline
\end{tabular}

Proof. (1) Since $N / K$ and $M^{+} / k$ are cyclic of degree 12 and 4 , respectively, then as in point (1) of Lemma 1 we obtain $\left(\zeta_{N} / \zeta_{M^{+}}\right)(s) \geq 0$ and $\left(\zeta_{M^{+}} / \zeta_{K}\right)(s) \geq 0$ for $0<s<1$.

(2) The first part follows from [Mar, Theorem III.1] or [LPL, Theorem 4]. For $K=\mathbb{Q}(\sqrt{2}, \sqrt{257})$ we have verified that $\zeta_{K}(s) \leq 0$ in the range $0<s<1$. Hence, $\zeta_{N}(s) \leq 0$ for $0<s<1$ for every $M$ with $h_{M}^{-}=1$. Since $N^{+} / k_{2}$ is abelian and $d_{N^{+}} / d_{k_{2}}^{12}=f^{16}$, using Proposition 2 we get upper bound $C$ on $f$ such that $h_{N}^{-}=1$ implies $f \leq C$. To alleviate the list of possible conductors $f$ we use the same argument as in point (3) of Lemma 1: If there is a prime divisor $l$ of $f$ which is inert in $\mathbb{Q}(\sqrt{p q})$, then $3^{4}$ divides $h_{N}^{-}$.

(3) Let $K_{12}$ be the compositum of $K$ and $K_{6}$. We have

$$
h_{N}^{-} / h_{M}^{-}=N_{\mathbb{Q}\left(\zeta_{12}\right) / \mathbb{Q}}\left(L(0, \chi) / 2^{4}\right) .
$$

Assume $\operatorname{Gal}(N / K)=\left\langle a, b^{2}\right\rangle$. Let $\chi_{-}$be any one of two quartic characters associated with the cyclic extension $M / K$ and $\chi_{+}$any one of two cubic characters associated with the cyclic extension $K_{12} / K$ such that $\chi=\chi_{-} \chi_{+}$. Using the Artin reciprocity theorem, it can be easily verified that $\chi_{-} \circ b=\chi_{-}$, $\chi_{-} \circ c=\chi_{-}^{-1}, \chi_{+} \circ b=\chi_{+}^{-1}$, and $\chi_{+} \circ c=\chi_{+}$, whence $\chi \circ b=\chi^{5}$ and $\chi_{\circ} \circ=\chi^{7}$. For a positive integer $n$ let $\sigma_{n} \in \operatorname{Gal}\left(\mathbb{Q}\left(\zeta_{12}\right) / \mathbb{Q}\right)$ with $\sigma_{n}\left(\zeta_{12}\right)=\zeta_{12}^{n}$. We have

$$
\sigma_{5}(L(0, \chi))=L\left(0, \chi^{5}\right)=L(0, \chi \circ b)=L(0, \chi)
$$

and $\sigma_{7}(L(0, \chi))=L(0, \chi)$. Since $\left\langle\sigma_{5}, \sigma_{7}\right\rangle=\operatorname{Gal}\left(\mathbb{Q}\left(\zeta_{12}\right) / \mathbb{Q}\right)$ we have $L(0, \chi) \in \mathbb{Q}$, whence $h_{N}^{-} / h_{M}^{-}$is the 4 -th power of some integer. 
Our computational results are given in Table 2 . When $K=\mathbb{Q}(\sqrt{2}, \sqrt{257})$, if $h_{N}=1$, then $N / M, N^{+} / M^{+}$, and $K_{6} / k_{2}$ are unramified. Otherwise, $h_{N} \equiv 0 \bmod 3$. Since $\mathbb{Q}(\sqrt{2})$ has class number one, we must have $k_{2}=\mathbb{Q}(\sqrt{257})$ and $(f)=1$. Note that when $K=\mathbb{Q}(\sqrt{5}, \sqrt{101}), k_{2}=\mathbb{Q}(\sqrt{101})$, and $f=2$, we have $K_{6}=\mathbb{Q}(\sqrt{101}, \theta)$ with $\theta^{3}-\theta^{2}-5 \theta-1=0$. Using $\mathrm{KASH}([\mathrm{K}])$ we verify that the class group of $\mathbb{Q}(\sqrt{5}, \sqrt{101}, \theta)$ is isomorphic to $C_{2}$ and the narrow class group of this field is isomorphic to $C_{4}$. It follows that $N^{+}$is the Hilbert class field of $\mathbb{Q}(\sqrt{5}, \sqrt{101}, \theta)$ and $N$ is the narrow Hilbert class field of this field. In addition, thanks to KASH we verify that the class number of $N^{+}$is equal to 1 .

4.2. $\operatorname{Gal}(M / \mathbb{Q})=Q_{8} \times C_{2}$. By $[$ Lou3, Theorem 1],

$$
M=\mathbb{Q}(\sqrt{-1}, \sqrt{2}, \sqrt{3}, \sqrt{-(2+\sqrt{2})(3+\sqrt{3})})
$$

is the only normal CM-field of relative class number one with Galois group isomorphic to $Q_{8} \times C_{2}$. This field has class number one and $Q_{M}=2$. In this subsection we assume that $N$ contains this field $M$ and will prove that $h_{N}>1$. The Galois $\operatorname{group} \operatorname{Gal}(N / \mathbb{Q})$ is isomorphic to either $Q_{8} \times C_{2} \times C_{3}$ or $Q_{24} \times C_{2}$ according to whether $N$ has a cyclic cubic subfield or not.

4.2.1. $\operatorname{Gal}(N / \mathbb{Q})=Q_{8} \times C_{2} \times C_{3}$. The field $N$ has only one cyclic cubic subfield $K_{3}$. The composita

$$
N_{1}=K_{3}(\sqrt{2}, \sqrt{3}, \sqrt{-(2+\sqrt{2})(3+\sqrt{3})}) \quad \text { and } \quad N_{2}=K_{3}(\sqrt{2}, \sqrt{3}, \sqrt{-1})
$$

have the same maximal real subfield $K_{3}(\sqrt{2}, \sqrt{3})$. Suppose that $h_{N}=1$. By Proposition 1(7) we would have $h_{N_{1}}^{-}=1$ or $h_{N_{2}}^{-}=1$. Since every octic quaternion CM-field has an even relative class number, $h_{N_{1}}^{-}$is even. Using [CK1] we verify that there is no imaginary abelian number field with Galois group isomorphic to $C_{2} \times$ $C_{2} \times C_{2} \times C_{3}$ of relative class number one which contains the field $\mathbb{Q}(\sqrt{2}, \sqrt{3}, \sqrt{-1})$. Hence $h_{N}>1$.

4.2.2. $\operatorname{Gal}(N / \mathbb{Q})=Q_{24} \times C_{2}$. The field $N$ contains a non-normal cubic subfield $K_{3}$. The compositum

$$
N_{1}=K_{3}(\sqrt{2}, \sqrt{3}, \sqrt{-(2+\sqrt{2})(3+\sqrt{3})})
$$

is a normal CM-field with Galois group isomorphic to $Q_{24}$. The compositum $N_{2}=$ $K_{3}(\sqrt{2}, \sqrt{3}, \sqrt{-1})$ is a normal CM-field with Galois group isomorphic to $D_{12} \times C_{2}$. We have $N=N_{1} N_{2}$ with $N_{1}^{+}=N_{2}^{+}=K_{3}(\sqrt{2}, \sqrt{3})$. Note that $h_{N_{1}}^{-}$is even. According to $\left[\mathrm{P}\right.$, Theorem 1], $h_{N_{2}}>1$. By Proposition 1(7) it follows that $h_{N}>1$.

$$
\text { 5. CAsE 3: } \operatorname{Gal}(M / \mathbb{Q}) \in\left\{G_{9}, G_{6}\right\}
$$

In subsection 5.1 we assume that $G(M / \mathbb{Q})=G_{9}$, and in subsection 5.2 we assume that $G(M / \mathbb{Q})=G_{6}$.

5.1. $\operatorname{Gal}(M / \mathbb{Q})=G_{9}$. There is only one normal CM-field $M$ of relative class number one with Galois group isomorphic to $G_{9}$ ([LO2, Theorem 20]):

$$
M=\mathbb{Q}(\sqrt{2}, \sqrt{5}, \sqrt{37}, \sqrt{-(2 \sqrt{2}+3 \sqrt{5})(2+\sqrt{5})}) .
$$


Assume that $N$ contains $M$. The aim of this subsection is to prove that $h_{N}>$ 1. Note that $\operatorname{Gal}(M / \mathbb{Q}(\sqrt{2 \cdot 5 \cdot 37}))=Q_{8}, \operatorname{Gal}(M / \mathbb{Q}(\sqrt{2}))=\operatorname{Gal}(M / \mathbb{Q}(\sqrt{5}))=$ $\operatorname{Gal}(M / \mathbb{Q}(\sqrt{37}))=D_{8}, \quad$ and $\operatorname{Gal}(M / \mathbb{Q}(\sqrt{2 \cdot 5}))=\operatorname{Gal}(M / \mathbb{Q}(\sqrt{2 \cdot 37}))=$ $\operatorname{Gal}(M / \mathbb{Q}(\sqrt{5 \cdot 37}))=C_{4} \times C_{2}$. Therefore, $\operatorname{Gal}(N / \mathbb{Q})$ is isomorphic to $G_{9} \times C_{3}$ if $N$ contains only one cubic cyclic subfield $K_{3},\left(Q_{8} \times C_{3}\right) \rtimes C_{2},\left(D_{8} \times C_{3}\right) \rtimes^{2} C_{2}$ $=\langle a, b, c| a^{3}=b^{2}=c^{2}=z^{4}=1, c^{-1} b c=b z^{2}, b z=z b, c z=z c, b^{-1} a b=a, c^{-1} a c=$ $\left.a, z^{-1} a z=a^{-1}\right\rangle$, or $\left(C_{4} \times C_{2} \times C_{3}\right) \rtimes C_{2}$ otherwise. We divide this subsection into four parts according to $\operatorname{Gal}(N / \mathbb{Q})$.

5.1.1. $\operatorname{Gal}(N / \mathbb{Q})=G_{9} \times C_{3}$. We will show that $h_{N}>1$. We first get an upper bound $C$ on the conductor of $K_{3} / \mathbb{Q}$ such that if $h_{N}^{-}=1$, then the conductor is less than or equal to $C$. Let $K=\mathbb{Q}(\sqrt{2}, \sqrt{5 \cdot 37})$. Since $N / K$ is cyclic of degree 12, then as in point (1) of Lemma 1 we obtain $\left(\zeta_{N} / \zeta_{M^{+}}\right)(s) \geq 0$ for $0<$ $s<1$. We verify that $\zeta_{M^{+}}(s) \leq 0$ for $\left.s \in\right] 0,1\left[\right.$, which implies $\zeta_{N}(s) \leq 0$ for $s \in] 0,1\left[\right.$. Let $F=\mathbb{Q}(\sqrt{2 \cdot 5 \cdot 37})$. The extension $N^{+} / F$ is abelian of degree 12 and $d_{N^{+}} / d_{F}^{12}=N_{F / \mathbb{Q}}\left(\mathfrak{F}_{F K_{3} / F}\right)^{8}$. Using $\mu_{F} \operatorname{Res}_{s=1}\left(\zeta_{F}\right) \leq 2.227842$ and Proposition 2, we obtain that if $h_{N}^{-} \leq 1$, then $N_{F / \mathbb{Q}}\left(\mathfrak{F}_{F K_{3} / F}\right) \leq 1300$, whence $\mathfrak{F}_{F K_{3} / F} \in$ $\left\{(7),(3)^{2},(13),(19),(31), \mathfrak{P}_{37}\right\}$ with $(37)=\mathfrak{P}_{37}^{2}$ in $F$.

(i) When $\mathfrak{F}_{F K_{3} / F}=(7), K_{3}$ is of conductor 7 . There are four non-normal octic CM-fields containing $\mathbb{Q}(\sqrt{2})$ with relative class number one. Let $M_{8,1}=$ $\mathbb{Q}(\sqrt{2}, \sqrt{5}, \sqrt{-(2 \sqrt{2}+3 \sqrt{5})(2+\sqrt{5})})$ and $M_{8.2}$ its conjugate over $\mathbb{Q}$. Set $N_{24,1}=M_{8,1} K_{3}$ and $N_{24,2}=M_{8,2} K_{3}$. By Proposition $1(5) h_{N}^{-}=\left(h_{N_{24,1}}^{-}\right)^{2}$. The two prime ideals lying above 7 in $M_{8,1}^{+}=\mathbb{Q}(\sqrt{2}, \sqrt{5})$ split in $M_{8,1}$. Hence, $3^{2} \mid h_{N_{24,1}}^{-}$by [LOO, Proposition 8] and $3^{4} \mid h_{N}^{-}$.

(ii) When $\mathfrak{F}_{F K_{3} / F}=(3)^{2}, K_{3}$ is of conductor 9. Let $M_{8,1}, M_{8,2}, N_{24,1}$, and $N_{24,2}$ be as in (i). One prime ideal lying above 37 in $\mathbb{Q}(\sqrt{2}, \sqrt{5})$ is ramified in $M_{8,1}$ and 37 splits in $K_{3}$, whence $2^{2} \mid h_{N_{24,1}}^{-}$and $2^{4} \mid h_{N}^{-}$. By the same argument we prove that if $\mathfrak{F}_{F K_{3} / F}=(19)$, then $2^{4} \mid h_{N}^{-}$.

(iii) When $\mathfrak{F}_{F K_{3} / F}=(13), K_{3}$ is of conductor 13. Let $M_{8,1}$ be any one of two nonnormal octic CM-fields of $M$ containing $\mathbb{Q}(\sqrt{2}, \sqrt{37})$ and $M_{8,2}$ its conjugate over $\mathbb{Q}$. Let $N_{24,1}=M_{8,1} K_{3}$ and $N_{24,2}=M_{8,2} K_{3}$. One prime ideal lying above 5 in $\mathbb{Q}(\sqrt{2}, \sqrt{37})$ is ramified in $M_{8,1}$ and 5 splits in $K_{3}$, which implies $2^{2} \mid h_{N_{24,1}}^{-}$and $2^{4} \mid h_{N}^{-}$.

(iv) When $\mathfrak{F}_{F K_{3} / F}=(31), K_{3}$ is of conductor 31 . We let $M_{8,1}$ be any one of two non-normal octic subfields of $M$ containing $\mathbb{Q}(\sqrt{5}, \sqrt{37})$ and $M_{8,2}$ its conjugate over $\mathbb{Q}$. One prime ideal lying above 2 is ramified in $M_{8,1}$ and 2 splits in $K_{3}$, whence $2^{2} \mid h_{N_{24,1}}^{-}$and $2^{4} \mid h_{N}^{-}$.

(v) When $\mathfrak{F}_{F K_{3} / F}=\mathfrak{P}_{37}, K_{3}$ is of conductor 37. According to [Ma], the cyclic sextic subfield $K_{3}(\sqrt{2 \cdot 5})$ of $N$ has class number 6 , whence by Proposition 1(6) $3 \mid h_{N}$.

5.1.2. $\operatorname{Gal}(N / \mathbb{Q})=\left(D_{8} \times C_{3}\right) \rtimes^{2} C_{2}$. Let $K_{3}$ be any one of three non-normal cubic subfields of $N, K_{6}$ its normal closure, and $k_{2}$ the quadratic subfield of $K_{6}$. We have $\operatorname{Gal}\left(M / k_{2}\right)=D_{8}$. Let $K$ be the intermediate field between $M^{+}$and $k_{2}$ such that $G(M / K)=C_{4}$. Then $M / K$ is unramified at all finite primes, $\operatorname{Gal}\left(M^{+} / k_{2}\right)=$ $C_{2} \times C_{2}, \operatorname{Gal}(N / K)=C_{12}$, and $\operatorname{Gal}\left(N^{+} / k_{2}\right)=C_{2} \times C_{2} \times C_{3}$. As in point (1) of 
TABLE 3 .

\begin{tabular}{|c|c|c|c|}
\hline$k$ & $f \leq$ & $f$ & $h_{N}^{-}$ \\
\hline $\mathbb{Q}(\sqrt{2})$ & 22 & None & \\
\hline $\mathbb{Q}(\sqrt{5})$ & 8 & None & \\
\hline $\mathbb{Q}(\sqrt{37})$ & 44 & 2 & $2^{4}$ \\
\hline
\end{tabular}

TABLE 4 .

\begin{tabular}{|c|c|c|c|}
\hline$k_{2}$ & $C$ & $f$ & $h_{N}^{-}$ \\
\hline $\mathbb{Q}(\sqrt{2 \cdot 5})$ & 140 & 37 & $1021^{2}$ \\
\hline $\mathbb{Q}(\sqrt{2 \cdot 37})$ & 21 & 9 & $5044^{2}$ \\
\hline $\mathbb{Q}(\sqrt{5 \cdot 37})$ & 35 & None & \\
\hline
\end{tabular}

Lemma 1 we obtain $\left(\zeta_{N} / \zeta_{M^{+}}\right)(s) \geq 0$ and $\zeta_{N}(s) \leq 0$ for $\left.s \in\right] 0,1\left[\right.$. Since $N^{+} / k_{2}$ is abelian of degree 12 and $d_{N^{+}} / d_{k_{2}}^{12}=f^{16} N_{K_{6} / \mathbb{Q}}\left(\mathfrak{D}_{N^{+} / K_{6}}\right)$, where $(f)=\mathfrak{F}_{K_{6} / k_{2}}$ and $\mathfrak{D}_{N^{+} / K_{6}}$ denotes the discriminant of the extension $N^{+} / K_{6}$, using Proposition 2 we obtain upper bound $C$ on $f$ such that if $h_{N}^{-}=1$, then $f \leq C$. Our computational results are given in Table 3 . As in point (3) of Lemma 2 we can easily verify that $h_{N}^{-} / h_{M}^{-}$is the 4 -th power of some rational integer.

5.1.3. $\operatorname{Gal}(N / \mathbb{Q})=\left(C_{4} \times C_{2} \times C_{3}\right) \rtimes C_{2}=\langle a, b, c, z| a^{3}=b^{2}=c^{2}=z^{4}=$ $\left.1, c^{-1} b c=b z^{2}, b z=z b, c z=z c, b^{-1} a b=a, c^{-1} a c=a^{-1}, z^{-1} a z=a\right\rangle$. In this case $N$ has three non-normal cubic subfields and its associated quadratic subfield $k_{2}$ has $\operatorname{Gal}\left(M / k_{2}\right)=C_{4} \times C_{2}$. For a fixed field $k_{2}$ there are two intermediate fields $K$ between $k_{2}$ and $M^{+}$such that $\operatorname{Gal}(M / K)=C_{4}$. Let $K$ be any one of these two fields. Then $M / K$ is unramified at all finite primes, $\operatorname{Gal}(N / K)=C_{12}$, $\operatorname{Gal}\left(M^{+} / k_{2}\right)=C_{2} \times C_{2}$, and $\operatorname{Gal}\left(N^{+} / k_{2}\right)=C_{2} \times C_{2} \times C_{3}$. Analogously as in subsection 5.1.2 we get upper bound $C$ on $f$ such that if $h_{N}^{-}=1$, then $f \leq C$. Since $\operatorname{Gal}\left(N / k_{2}\right)=C_{4} \times C_{2} \times C_{3}$, we compute $h_{N}^{-}$using Hecke $L$-functions over $k_{2}$ (see Table 4).

5.1.4. $\operatorname{Gal}(N / \mathbb{Q})=\left(Q_{8} \times C_{3}\right) \rtimes C_{2}$. In this case $N$ has three non-normal cubic subfields such that its associated quadratic subfield $k_{2}$ is equal to $\mathbb{Q}(\sqrt{2 \cdot 5 \cdot 37})$. Let $K$ be any one of three quartic fields containing $\mathbb{Q}(\sqrt{2 \cdot 5 \cdot 37})$. Then $\operatorname{Gal}(N / K)=$ $C_{12}$ and $\operatorname{Gal}\left(N^{+} / k_{2}\right)=C_{2} \times C_{2} \times C_{3}$. As in subsection 5.1.2 we verify that if $h_{N}^{-}=1$, then $f \leq 36$ with $\mathfrak{F}_{K_{6} / k_{2}}=(f)$. There is no sextic field $K_{6}$ containing $\mathbb{Q}(\sqrt{2 \cdot 5 \cdot 37})$ with $f \leq 36$. Therefore, $h_{N}^{-}>1$.

5.2. $\operatorname{Gal}(M / \mathbb{Q})=G_{6}$. In Lou3] it is proved that there are exactly two such fields $M$ with $h_{M}=1$ : the composita $M=M_{1} M_{2}$ listed in Table 5 . In fact, those are the only fields with Galois group isomorphic to $G_{6}$ of relative class number one. The Galois group $\operatorname{Gal}\left(M^{+} / \mathbb{Q}\right)$ is isomorphic to $D_{8}$ or $C_{4} \times C_{2}$. When $\operatorname{Gal}\left(M^{+} / \mathbb{Q}\right)=D_{8}$, $M$ is a compositum of an octic dihedral CM-field $M_{8,1}$ and an imaginary abelian number field $M_{8,2}$ with $\operatorname{Gal}\left(M_{8,2} / \mathbb{Q}\right)=C_{4} \times C_{2}$. Using [YK] and [CK1], we verify 
TABLE 5 .

\begin{tabular}{|c|c|c|c|}
\hline$M_{8,1}$ & $M_{8,2}$ & $h_{M_{8,1}}^{-}$ & $h_{M_{8,2}}^{-}$ \\
\hline $\mathbb{Q}(\sqrt{2}, \sqrt{17}, \sqrt{-(5+\sqrt{17}) / 2})$ & $\mathbb{Q}(\sqrt{2}, \sqrt{17}, \sqrt{-(17+3 \sqrt{17}) / 2})$ & 1 & 2 \\
$\operatorname{Gal}\left(M_{8.1} / \mathbb{Q}\right)=D_{8}$ & $\operatorname{Gal}\left(M_{8.2} / \mathbb{Q}\right)=D_{8}$ & & \\
$\operatorname{Gal}\left(M_{8,1} / \mathbb{Q}(\sqrt{34})\right)=C_{4}$ & $\operatorname{Gal}\left(M_{8,2} / \mathbb{Q}(\sqrt{2})\right)=C_{4}$ & & \\
\hline $\mathbb{Q}(\sqrt{13}, \sqrt{17}, \sqrt{-(9+\sqrt{13}) / 2})$ & $\mathbb{Q}(\sqrt{17}, \sqrt{-(13+2 \sqrt{13}}))$ & 1 & 2 \\
$\operatorname{Gal}\left(M_{8.1} / \mathbb{Q}\right)=D_{8}$ & $\operatorname{Gal}\left(M_{8.2} / \mathbb{Q}\right)=C_{4} \times C_{2}$ & & \\
$\operatorname{Gal}\left(M_{8,1} / \mathbb{Q}(\sqrt{221})\right)=C_{4}$ & $\operatorname{Gal}(\mathbb{Q}(\sqrt{-(13+2 \sqrt{13}}) / \mathbb{Q})=C_{4}$ & & \\
\hline
\end{tabular}

that there is only one such field $M$ with $h_{M}^{-}=1$ : the second field in Table 5 . When $\operatorname{Gal}\left(M^{+} / \mathbb{Q}\right)=C_{4} \times C_{2}, M$ is a compositum of two octic dihedral CM-fields $M_{8,1}$ and $M_{8,2}$ with $M_{8,1}^{+}=M_{8,2}^{+}$. According to [Lou3, Theorem 2] there is only one such field $M$ with $h_{M}^{-}=1$ : the first field in Table 5 . We will prove that $h_{N}>1$. If $K_{3} / \mathbb{Q}$ is normal, then $\operatorname{Gal}(N / \mathbb{Q})=G_{6} \times C_{3}$. Otherwise, $\operatorname{Gal}(N / \mathbb{Q})=C_{3} \rtimes G_{6}$. Let $N_{1}=M_{8,1} K_{3}$ and $N_{2}=M_{8,2} K_{3}$. Then $N=N_{1} N_{2}$ with $N_{1}^{+}=N_{2}^{+}=K_{3} K_{4}$.

5.2.1. $\operatorname{Gal}(N / \mathbb{Q})=G_{6} \times C_{3}$. If $\operatorname{Gal}\left(M^{+} / \mathbb{Q}\right)=C_{4} \times C_{2}$, then $\operatorname{Gal}\left(N_{1} / \mathbb{Q}\right)=D_{8} \times$ $C_{3}=\operatorname{Gal}\left(N_{2} / \mathbb{Q}\right)$. According to $\left[\mathrm{P}\right.$, Theorem 1], $h_{N_{1}}^{-}>1$ and $h_{N_{2}}^{-}>1$, whence $h_{N}>1$. If $\operatorname{Gal}\left(M^{+} / \mathbb{Q}\right)=D_{8}$, then $\operatorname{Gal}\left(N_{1} / \mathbb{Q}\right)=D_{8} \times C_{3}$, and $\operatorname{Gal}\left(N_{2} / \mathbb{Q}\right)=$ $C_{4} \times C_{2} \times C_{3}$. Using [CK1], we verify that $h_{N_{2}}^{-}>4$, whence $h_{N}^{-}>1$.

\subsection{2. $\operatorname{Gal}(N / \mathbb{Q})=C_{3} \rtimes G_{6}$.}

(a) Let $M$ be the first field in Table 5 . The quadratic field $k_{2}$ associated with $K_{3}$ is either $\mathbb{Q}(\sqrt{34}), \mathbb{Q}(\sqrt{2})$, or $\mathbb{Q}(\sqrt{17})$. If $k_{2}=\mathbb{Q}(\sqrt{34})$, then $\operatorname{Gal}\left(N_{1} / \mathbb{Q}\right)=D_{24}$, and $\operatorname{Gal}\left(N_{2} / \mathbb{Q}\right)=C_{3} \rtimes D_{8}$. If $k_{2}=\mathbb{Q}(\sqrt{2})$, then $\operatorname{Gal}\left(N_{1} / \mathbb{Q}\right)=C_{3} \rtimes D_{8}$, and $\operatorname{Gal}\left(N_{2} / \mathbb{Q}\right)=D_{24}$. If $k_{2}=\mathbb{Q}(\sqrt{17})$, then $\operatorname{Gal}\left(N_{1} / \mathbb{Q}\right)=C_{3} \rtimes D_{8}=$ $\operatorname{Gal}\left(N_{2} / \mathbb{Q}\right)$. According to $[\mathrm{P}$, Theorem 1] and [Lef, Theorem 4.1], we have $h_{N_{1}}^{-}>1$ and $h_{N_{2}}^{-}>1$, whence $h_{N}>1$.

(b) Let $M$ be the second field in Table 5. The quadratic field $k_{2}$ associated with $K_{3}$ is either $\mathbb{Q}(\sqrt{221}), \mathbb{Q}(\sqrt{13})$, or $\mathbb{Q}(\sqrt{17})$. If $k_{2}=\mathbb{Q}(\sqrt{221})$, then $\operatorname{Gal}\left(N_{1} / \mathbb{Q}\right)=D_{24}$, and $\operatorname{Gal}\left(N_{2} / \mathbb{Q}\right)=S_{3} \times C_{4}$. If $k_{2}=\mathbb{Q}(\sqrt{13})$, then $\operatorname{Gal}\left(N_{1} / \mathbb{Q}\right)=C_{3} \rtimes D_{8}$ and $\operatorname{Gal}\left(N_{2} / \mathbb{Q}\right)=Q_{12} \times C_{2}$. If $k_{2}=\mathbb{Q}(\sqrt{17})$, then $\operatorname{Gal}\left(N_{1} / \mathbb{Q}\right)=C_{3} \rtimes D_{8}$, and $\operatorname{Gal}\left(N_{2} / \mathbb{Q}\right)=S_{3} \times C_{4}$. Using [Lef, Theorem 4.1] and [ $\mathrm{P}$, Theorem 1], we have $h_{N_{1}}^{-}>1$ and $h_{N_{2}}^{-}>1$, whence $h_{N}>1$.

\section{6. $\operatorname{CASE} 4: \operatorname{Gal}(M / \mathbb{Q})=D_{8} \times C_{2}$}

To begin with, we prove the following:

Theorem 2 (Compare with [Lou3 Theorems 2 and 3]). There are four normal $C M$-fields $M$ of degree 16 and Galois group $D_{8} \times C_{2}$ with relative class number one, those given in the following Table 6 . 
TABLE 6 .

\begin{tabular}{|c|c|c|c|}
\hline$M^{+}$ & $\alpha: M=M^{+}(\sqrt{-\alpha})$ & $Q_{M}$ & $\omega_{M}$ \\
\hline $\mathbb{Q}(\sqrt{2}, \sqrt{5}, \sqrt{17})$ & $5(5+\sqrt{17}) / 2$ & 2 & 2 \\
\hline $\mathbb{Q}(\sqrt{2}, \sqrt{3}, \sqrt{3+\sqrt{3}})$ & 1 & 2 & 24 \\
$\mathbb{Q}(\sqrt{3}, \sqrt{11}, \sqrt{15+8 \sqrt{3}})$ & 1 & 2 & 12 \\
$\mathbb{Q}(\sqrt{2}, \sqrt{17}, \sqrt{3(5+\sqrt{17}) / 2})$ & 3 & 2 & 6 \\
\hline
\end{tabular}

Proof. The Galois group $\operatorname{Gal}\left(M^{+} / \mathbb{Q}\right)$ is isomorphic to either $D_{8}$ or $C_{2} \times C_{2} \times C_{2}$. When $\operatorname{Gal}\left(M^{+} / \mathbb{Q}\right)=D_{8}, M$ is a compositum of an octic dihedral CM-field $M_{8,1}$ and an imaginary abelian number field $M_{8,2}$ with $\operatorname{Gal}\left(M_{8,2} / \mathbb{Q}\right)=C_{2} \times C_{2} \times C_{2}$. Let $L$ be any one of four non-normal quartic CM-subfields of $M_{8,1}$. According to Lou3. Proposition 16], $h_{M}^{-}=1$ if and only if $\left(h_{L}^{-}, h_{M_{8,2}}^{-}\right) \in\{(1,4),(1,2),(2,1)\}$. In the case that $\left(h_{L}^{-}, h_{M_{8,2}}^{-}\right)=(1,4)$ or $(1,2), M_{8,1}^{+}=M_{8,2}^{+}$is of the form $\mathbb{Q}(\sqrt{p}, \sqrt{q})$, where $(p, q)$ is one of the 19 pairs given in [LO1, Theorem 8]. Then $M_{8,2}=$ $\mathbb{Q}(\sqrt{p}, \sqrt{q}, \sqrt{-m})$, where $\mathbb{Q}(\sqrt{-m})$ is one of $81(=9+18+54)$ imaginary quadratic fields of class number one, two or four. For these $1539(=19 \times 81)$ CM-fields $M_{8,2}$ we compute $h_{M_{8,2}}^{-}$and verify that there is only one field $M$ with $h_{M}^{-}=1$ : the fourth field in Table 6 . In the case that $\left(h_{L}^{-}, h_{M_{8,2}}^{-}\right)=(2,1)$, using [YK] and [CK1] we verify that there are exactly two fields $M$ with $h_{M}^{-}=1$ : the second and third fields in Table 6. When $\operatorname{Gal}\left(M^{+} / \mathbb{Q}\right)=C_{2} \times C_{2} \times C_{2}, M$ is a compositum of two octic dihedral CM-fields $M_{8,1}$ and $M_{8,2}$. According to [Lou3 Theorem 2], there is only one such $M$ with $h_{M}^{-}=1$ : the first field in Table 6 .

From now on we assume that $M$ is one of these four fields and we will prove that $h_{N}>1$. We classify the Galois group $\operatorname{Gal}(N / \mathbb{Q})$. Let $K_{3}$ be any cubic subfield of $N$. If $K_{3}$ is not normal, then its normal closure $K_{6}$ is a dihedral real sextic field and we let $k_{2}$ denote the (real) quadratic subfield of $K_{6}$. If $K_{3}$ is normal, then $\operatorname{Gal}(N / \mathbb{Q})=D_{8} \times C_{6}$. If $K_{3}$ is not normal over $\mathbb{Q}$, then $\operatorname{Gal}(N / \mathbb{Q})$ is isomorphic to either $D_{8} \times S_{3}, D_{24} \times C_{2}$, or $\left(C_{3} \rtimes D_{8}\right) \times C_{2}$. Let $K_{4}=M_{8,1}^{+}=M_{8,2}^{+}$. If $K_{4} \cap k_{2}=\mathbb{Q}$, then $\operatorname{Gal}\left(M^{+} / \mathbb{Q}\right)=C_{2} \times C_{2} \times C_{2}$, and $\operatorname{Gal}(N / \mathbb{Q})=D_{8} \times S_{3}$. If $K_{4} \cap k_{2} \supsetneqq \mathbb{Q}$, then $M_{8,1} / k_{2}$ is either cyclic or biquadratic bicyclic. If $M_{8,1} / k_{2}$ is cyclic quartic, then $\operatorname{Gal}(N / \mathbb{Q})=D_{24} \times C_{2}$. If $M_{8,1} / k_{2}$ is biquadratic bicyclic, then $\operatorname{Gal}\left(K_{3} M_{8,1} / \mathbb{Q}\right)=$ $C_{3} \rtimes D_{8}=\left\langle a, b, c \mid a^{3}=b^{4}=c^{2}=1, b^{-1} a b=a^{-1}, c^{-1} a c=a, c^{-1} b c=b^{-1}\right\rangle$, and $\operatorname{Gal}(N / \mathbb{Q})=\left(C_{3} \rtimes D_{8}\right) \times C_{2}$.

6.1. $\operatorname{Gal}(N / \mathbb{Q})=D_{8} \times C_{6}$. If $\operatorname{Gal}\left(M^{+} / \mathbb{Q}\right)=D_{8}$, then the composita $N_{1}=M_{8,1} K_{3}$ and $N_{2}=M_{8,2} K_{3}$ are normal CM-subfields of $N$ with the same maximal real subfields $K_{3} K_{4}$. By [P, Theorem 1], the compositum of the dihedral octic CM-field $\mathbb{Q}(\sqrt{13}, \sqrt{17}, \sqrt{-(9+\sqrt{13}) / 2})$ and of the cyclic cubic field of conductor 13 is the only normal CM-field of relative class number one with Galois group isomorphic to $D_{8} \times C_{3}$. In Table 6 there is no field $M$ containing $\mathbb{Q}(\sqrt{13}, \sqrt{17})$. It follows then that $h_{N_{1}}^{-}>1$. According to [CK1], there are exactly two imaginary abelian number fields with Galois group isomorphic to $C_{2} \times C_{2} \times C_{2} \times C_{3}$ of relative class number one: $F_{7}(\sqrt{-1}, \sqrt{-3}, \sqrt{-7})$ and $F_{7}(\sqrt{-3}, \sqrt{-7}, \sqrt{-15})$, where $F_{7}$ denotes the cyclic 
cubic field of conductor 7 . In Table 6 there is no field $M$ containing $\mathbb{Q}(\sqrt{3}, \sqrt{7})$, or $\mathbb{Q}(\sqrt{5}, \sqrt{21})$, whence $h_{N_{2}}^{-}>1$. It follows that if $\operatorname{Gal}\left(M^{+} / \mathbb{Q}\right)=D_{8}$, then $h_{N}>1$. If $\operatorname{Gal}\left(M^{+} / \mathbb{Q}\right)=C_{2} \times C_{2} \times C_{2}$, then $N_{1}=M_{8,1} K_{3}$ and $N_{2}=M_{8,2} K_{3}$ are normal CM-fields with Galois group isomorphic to $D_{8} \times C_{3}$. Note that $M_{8,1}^{+}=M_{8,2}^{+}=$ $\mathbb{Q}(\sqrt{2}, \sqrt{17})$. According to $[\mathrm{P}$, Theorem 1$], h_{N_{1}}^{-}>1$ and $h_{N_{2}}^{-}>1$, which implies $h_{N}>1$.

6.2. $\operatorname{Gal}(N / \mathbb{Q})=D_{8} \times S_{3}$. In this case $N$ has three non-normal real cubic subfields. Let $K_{3}, K_{6}, k_{2}$ and $K_{4}$ be as above. We have that $K_{4} \cap k_{2}=\mathbb{Q}, \operatorname{Gal}\left(M^{+} / \mathbb{Q}\right)=$ $C_{2} \times C_{2} \times C_{2}, M$ is the first field in Table 6 . In addition, we have $K_{4}=\mathbb{Q}(\sqrt{2}, \sqrt{17})$, and $k_{2}=\mathbb{Q}(\sqrt{m})$ with $m \in\{5,2 \cdot 5,5 \cdot 17,2 \cdot 5 \cdot 17\}$. Let $(f)$ be the conductor of the extension $K_{6} / k_{2}$ with $f$ a positive integer.

Lemma 3. 1. We have $\zeta_{N}(s) \leq 0$ for $0<s<1$.

2. For each given $k_{2}$ in the above we can compute a bound of $f \leq C$ on the conductor $(f)$ for $N$ 's such that $h_{N}^{-}=1$. These bounds and the possible $f$ 's are compiled in Table 7.

3. The quotient $h_{N}^{-} / h_{M}^{-}$is the perfect fourth power of some rational integer.

Proof. (1) Let $\chi_{N / M^{+}}$be any one of two characters associated with the cyclic sextic extension $N / M^{+}$. We have

$$
\frac{\zeta_{N}(s)}{\zeta_{M^{+}}(s)}=\frac{\zeta_{M}(s)}{\zeta_{M^{+}}(s)}\left|L\left(s, \chi_{N / M^{+}}\right) L\left(s, \chi_{N / M^{+}}^{2}\right)\right|^{2}
$$

and

$$
\frac{\zeta_{M}(s)}{\zeta_{M^{+}}(s)}=\frac{\zeta_{M_{8,1}}(s)}{\zeta_{K_{4}}(s)} \frac{\zeta_{M_{8,2}}(s)}{\zeta_{K_{4}}(s)}=L\left(s, \psi_{1}\right)^{2} L\left(s, \psi_{2}\right)^{2},
$$

where $\psi_{i}$ is the unique irreducible character of degree 2 of $\operatorname{Gal}\left(M_{8 . i} / \mathbb{Q}\right)$ the dihedral group of order 8 , and $L\left(s, \psi_{i}\right)$ denotes the Artin $L$-function associated with $\psi_{i}$ for $i=1,2$. Since $\psi_{i}$ is real valued, $L\left(s, \psi_{i}\right)$ is on the real axis and $L\left(s, \psi_{i}\right)^{2} \geq 0$. For $M^{+}=\mathbb{Q}(\sqrt{2}, \sqrt{5}, \sqrt{17})$ we have verified that $\zeta_{M^{+}}(s) \leq 0$ for $s \in] 0,1\left[\right.$, whence $\zeta_{N}(s) \leq 0$.

(2) Since $M / M^{+}$is unramified at all finite primes, $N / N^{+}$is unramified at all finite primes and $d_{N} / d_{N^{+}}=d_{N^{+}}=d_{k_{2}}^{12} f^{16} N_{K_{6} / \mathbb{Q}}\left(\mathfrak{D}_{N^{+} / K_{6}}\right)$. Using Proposition 2, we get an upper bound on $f$. Since the prime ideals lying above 2 and those above 17 split in $M / M^{+}$, if $(f, 2)>1$ or $(f, 17)>1$, then $3^{2}$ divides $h_{N}^{-}$ by [LOO, Proposition 8]. Note that the prime ideals lying above 13 split in $M / M^{+}$, whence the relative class number of the fourth field $N$ in Table 7 is divisible by $3^{4}$.

TABLE 7 .

\begin{tabular}{|c|c|c|c|c|}
\hline$k_{2}$ & $\mu_{k_{2}} \operatorname{Res}_{s=1}\left(\zeta_{k_{2}}\right) \leq$ & $f \leq$ & $f$ & $h_{N}^{-}$ \\
\hline $\mathbb{Q}(\sqrt{5})$ & 0.0436324 & 10 & NONE & \\
\hline $\mathbb{Q}(\sqrt{2 \cdot 5})$ & 0.4276490 & 41 & 37 & $(920)^{4}$ \\
\hline $\mathbb{Q}(\sqrt{5 \cdot 17})$ & 0.5861712 & 31 & 9 & $(44)^{4}$ \\
\hline $\mathbb{Q}(\sqrt{2 \cdot 5 \cdot 17})$ & 1.4062136 & 38 & 13 & $3^{4} \mid h_{N}^{-}$ \\
\hline
\end{tabular}


(3) Note that $M_{8,1}$ and $M_{8,2}$ are cyclic over $\mathbb{Q}(\sqrt{34})$. Let $K$ be the compositum of $\mathbb{Q}(\sqrt{34})$ and $k_{2}$. Then $\operatorname{Gal}(N / K)=C_{12}$. Let $\chi$ be any one of the four characters of order 12 associated with the cyclic extension $N / K$. Similarly to point (3) of Lemma 2 , we verify that $L(0, \chi) \in \mathbb{Q}$ and $h_{N}^{-} / h_{M}^{-}=\left(L(0, \chi) / 2^{4}\right)^{4}$.

In conclusion, we have proved that every normal CM-field with Galois group isomorphic to $D_{8} \times S_{3}$ has class number greater than one. Our computational results are given in Table 7 .

6.3. $\operatorname{Gal}(N / \mathbb{Q})=D_{24} \times C_{2}$. In this case $N$ has three non-normal cubic fields and $M_{8,1} / k_{2}$ is cyclic. Let $N_{1}=M_{8,1} K_{3}$ and $N_{2}=M_{8,2} K_{3}$. Then we have $N=N_{1} N_{2}$ with $N_{1}^{+}=N_{2}^{+}=K_{3} K_{4}$. If $\operatorname{Gal}\left(M^{+} / \mathbb{Q}\right)=D_{8}$, then $\operatorname{Gal}\left(N_{1} / \mathbb{Q}\right)=D_{24}$, and $\operatorname{Gal}\left(N_{2} / \mathbb{Q}\right)=S_{3} \times C_{2} \times C_{2}$. If $\operatorname{Gal}\left(M^{+} / \mathbb{Q}\right)=C_{2} \times C_{2} \times C_{2}$, then $\operatorname{Gal}\left(N_{1} / \mathbb{Q}\right)=$ $D_{24}=\operatorname{Gal}\left(N_{2} / \mathbb{Q}\right)$. Using [Lef, Theorem 4.1] and [P Theorem 1], we verify that in both cases $h_{N_{1}}^{-}>1$ and $h_{N_{2}}^{-}>1$. It follows that the class number of a normal CM-field with Galois group isomorphic to $D_{24} \times C_{2}$ is greater than one.

6.4. $\operatorname{Gal}(N / \mathbb{Q})=\left(C_{3} \rtimes D_{8}\right) \times C_{2}$. In this case $N$ has three non-normal cubic fields and $M_{8,1} / k_{2}$ is biquadratic bicyclic. Then the Galois group of the compositum $N_{1}=M_{8,1} K_{3}$ over $\mathbb{Q}$ is isomorphic to $C_{3} \rtimes D_{8}$, whence $h_{N_{1}}^{-}>1$ ([ᄑ Theorem 13]). If $\operatorname{Gal}\left(M^{+} / \mathbb{Q}\right)=D_{8}$, then $\operatorname{Gal}\left(N_{2} / \mathbb{Q}\right)=S_{3} \times C_{2} \times C_{2}$. If $\operatorname{Gal}\left(M^{+} / \mathbb{Q}\right)=C_{2} \times C_{2} \times C_{2}$, then $\operatorname{Gal}\left(N_{2} / \mathbb{Q}\right)=C_{3} \rtimes D_{8}$. By $\left[\mathbb{P}\right.$, Theorems 1 and 13] $h_{N_{2}}^{-}>1$. Consequently, if $\operatorname{Gal}(N / \mathbb{Q})=\left(C_{3} \rtimes D_{8}\right) \times C_{2}$, then $h_{N}>1$.

To conclude, Theorem 1 is now proved with completion.

All computations were carried out using Pari-Gp $([\mathrm{Pa}])$ and KASH $([\underline{\mathrm{K}}])$.

\section{ACKNOWLEDGMENTS}

This research was supported by Grant BK21.

\section{REFERENCES}

[CH] P. E. Conner and J. Hurrelbrink, Class number parity, Series in Pure Mathematics, Vol. 8, World Scientific, 1988. MR 90f:11092

[CK1] K.-Y. Chang and S.-H. Kwon, Class numbers of imaginary abelian number fields, Proc. Amer. Math. Soc., 128 (2000), 2517-2528. MR 2000m:11108

[CK2] K.-Y. Chang and S.-H. Kwon, CM-fields of degree 2pq, preprint.

[CK3] K.-Y. Chang and S.-H. Kwon, The non-abelian normal CM-fields of degree 36 with class number one, Acta Arith., 101 (2002), 53-61.

$[\mathrm{H}] \quad J$. Hoffstein, Some analytic bounds for zeta functions and class numbers, Invent. Math., 55 (1979), 37-47. MR 80k:12019

[Ho] K. Horie, On a ratio between relative class numbers, Math. Z., 211 (1992), 505-521. MR 94a:11171

[JL] G. James and M. Liebeck, Representations and characters of groups, Cambridge Mathematical Textbooks, Cambridge University Press, 1993. MR 94h:20007

[K] M. Daberkow, C. Fieker, J. Klüners, M. Phost, K. Roegner and K. Wildanger, Kant $V_{4}$, J. Symbolic Comput. 24 (1997), 267-283. MR 99g:11150

[Lef] Y. Lefeuvre, Corps diédreaux à multiplication complexe principaux, Ann., Inst. Fourier, 50 (2000), 67-103. MR 2001g:11166

[LLO] F. Lemmermeyer, S. Louboutin, et R. Okazaki, The class number one problem for some non-abelian normal CM-fields of degree 24, J. Théor. Nombres Bordeaux 11 (1999), 387406. MR 2001j:11104

[LO1] S. Louboutin and R. Okazaki, Determination of all non-normal quartic CM-fields and of all non-abelian normal octic CM-fields with class number one, Acta Arith., 67 (1994), 47-62. MR 95g:11107 
[LO2] S. Louboutin and R. Okazaki, The class number one problem for some non-abelian normal CM-fields of 2-power degrees, Proc. London Math. Soc., (3) 76 (1998), 523-548. MR 99c:11138

[LOO] S. Louboutin, R. Okazaki and M. Olivier, The class number one problem for some non-abelian normal CM-fields, Trans., Amer. Math. Soc. 349 (1997), 3657-3678. MR 97k:11149

[Lou1] S. Louboutin, Determination of all quaternion octic CM-fields with class number 2, J. London Math. Soc., (2) 54 (1996), 227-238. MR 97g:11122

[Lou2] S. Louboutin, Upper bounds on $|L(1, \chi)|$ and applications, Canad. J. Math., 50 (1998), 794-815. MR 99f: 11138

[Lou3] S. Louboutin, The class number one problem for the non-abelian normal CM-fields of degree 16, Acta Arith., 82 (1998), 173-196. MR 98j:11097

[Lou4] S. Louboutin, The class number one problem for the dihedral and dicyclic CM-fields, Colloq. Math. 80 (1999), 259-265. MR 2000e: 11140

[Lou5] S. Louboutin, Computation of relative class numbers of CM-fields by using Hecke Lfunctions, Math. Comp., 69 (2000), 371-393. MR 2000i:11172

[Lou6] S. Louboutin, Computation of $L(0, \chi)$ and of relative class numbers of CM-fields, Nagoya Math. J., 161 (2001), 171-191. MR 2002e:11151

[LP1] S. Louboutin and Y.-H. Park, Class number problems for dicyclic CM-fields, Publ. Math. Debrecen, 57 (2000), 283-295. MR 2001m:11196

[LP2] S. Louboutin and Y.-H. Park, The Class number one problem for the non-abelian normal CM-fields of degree 42, preprint.

[LPL] S. Louboutin, Y.-H. Park and Y. Lefeuvre, Construction of the real dihedral number fields of degree 2p. Applications, Acta Arith. 89 (1999), 201-215. MR 2000g:11101

[M] J. M. Masley, Class numbers of real cyclic number fields with small conductor, Compositio Math. 37 (1978), 297-319. MR 80e:12005

[Ma] S. Mäki, The determination of units in real cyclic sextic fields, Lecture Notes in Math., Vol. 797 (1980), Springer-Verlag. MR 82a:12004

[Mar] J. Martinet, Sur l'arithmétique des extensions galoisiennes à groupe de Galois diedral d'ordre $2 p$, Ann. Inst. Fourier (Grenoble) 19 (1969), 1-80. MR 41:6820

[O] A. M. Odlyzko, Some analytic estimates of class numbers and discriminants, Invent. Math., 29 (1975), 279-286. MR 51:12788

[Ok] R. Okazaki, Inclusion of CM-fields and divisibility of relative class numbers, Acta Arith. 92 (2000), 319-338. MR 2001h:11138

[P] Y.-H. Park, The class number one problem for the non-abelian normal CM-fields of degree 24 and 40, Acta Arith., 101 (2002), 63-80.

[Pa] C. Batut, K. Belabas, D. Bernardi, H. Cohen, M. Olivier, Pari-GP version 2.0.11.

[PK] Y.-H. Park and S.-H. Kwon, Determination of all non-quadratic imaginary cyclic number fields of 2-power degree with relative class number $\leq 20$, Acta Arith., 83 (1998), 211-223. MR 99a: 11125

[PsK] S.-M. Park and S.-H. Kwon, The class number one problem for normal CM-fields of degree 32 II, preprint.

[W] L. Washington, Introduction to cyclotomic fields, 2nd ed., Grad. Texts in Math., Vol. 83, Springer-Verlag, 1997. MR 97h:11130

[Y] K. Yamamura, The determination of the imaginary abelian number fields with class number one, Math. Comp. 62 (1994), 899-921. MR 94g:11096

[YK] H.-S. Yang and S.-H. Kwon, The non-normal quartic CM-fields and the octic dihedral CM-fields with relative class number two, J. Number Theory 79 (1999), 175-193. MR 2000h:11117

[YPK] H.-S. Yang, S.-M. Park and S.-H. Kwon, Class number one problem for normal CM-fields of degree 32, preprint.

Information Security Basic Research Team, Etri, 161 Kajong-dong, Yusong-Gu, 305-350, TAEJON, KorEA

E-mail address: jang1090@etri.re.kr

Department of Mathematics Education, Korea University, 136-701, Seoul, Korea

E-mail address: shkwon@semi.korea.ac.kr 